Ciência Florestal, Santa Maria, v. 23, n. 2, p. 529-536, abr.-jun., 2013

ISSN 0103-9954

\title{
PREDICCIÓN BAYESIANA DE PARÁMETROS GENÉTICOS EN CLONES DE Eucalyptus globulus BAJO CONDICIONES DE SUPLEMENTO HÍDRICO
}

\section{BAYESIAN PREDICTION OF GENETIC PARAMETERS IN Eucalyptus globulus CLONES UNDER WATER SUPPLY CONDITIONS}

\author{
Freddy Mora $^{1}$ Rafael Rubilar ${ }^{2} \quad$ Veronica Ingrid Emhart ${ }^{3} \quad$ Javier Saavedra $^{4}$
}

\begin{abstract}
RESUMEN
Se realizó un análisis Bayesiano de parámetros genéticos del crecimiento en 29 clones de Eucalyptus globulus de doce meses de edad, en el sur de Chile. Se consideraron dos condiciones ambientales contrastantes en cuanto a la disponibilidad hídrica: 1. Sin irrigación, y 2. Las plantas se irrigaron con un sistema localizado de riego. Para el análisis Bayesiano se utilizó el algoritmo de Gibbs en un modelo de interacción clonambiente. Los valores de heredabilidad fueron altos en la condición de riego (moda a posteriori $\mathrm{de}^{2}=0,41$, 0,36 y 0,39 para la altura, diámetro y área basal, respectivamente), en tanto que en la situación sin riego, los valores de las heredabilidades fueron significativamente más bajos; confirmado por medio de los intervalos de credibilidad Bayesianos (95\% de probabilidad). La moda a posteriori de la correlación genética entre sitios fue positiva y alta para las tres características $(\mathrm{r}=0,7,0,65$ y 0,8 , para altura, diámetro y área basal, respectivamente), y de acuerdo al intervalo de credibilidad, esta correlación fue estadísticamente diferente de cero, indicando una interacción no significativa.
\end{abstract}

Palabras-clave: riego; crecimiento; heredabilidad; valores genéticos.

\begin{abstract}
A Bayesian analysis of genetic parameters for growth traits at twelve months after planting was carried out in twenty nine Eucalyptus globulus clones in southern Chile. Two different environmental conditions were considered: 1) Non-irrigation and; 2) Plants were irrigated with a localized irrigation system. The Bayesian approach was performed using Gibbs sampling algorithm in a clone-environment interaction model. Heritability values were high in the water supply condition (posterior mode: $\mathrm{H} 2=0.41,0.36$ and 0.39 for height, diameter and sectional area, respectively), while in the environment without irrigation, the heritabilities were significantly lower, which was confirmed by the Bayesian credible intervals ( $95 \%$ probability). The posterior mode of the genetic correlation between sites was positive and high for all traits $(\mathrm{r}=0.7$, 0.65 and 0.8 , for height, diameter and sectional area, respectively) and according to the credible interval, it was statistically different from zero, indicating a non-significant interaction.
\end{abstract}

Keywords: irrigation; growth traits; heritability; breeding values.

1. Ingeniero Forestal, MSc., Dr., Profesor del Instituto de Biología Vegetal y Biotecnología, Universidad de Talca, 2 Norte 685. Talca, Chile. morapoblete@gmail.com

2. Ingeniero Forestal, MSc., Dr., Profesor del Departamento de Silvicultura, Facultad de Ciencias Forestales, Universidad de Concepción, Victoria 631, Barrio Universitario, Concepción, Chile. rafaelrubilar@udec.cl

3. Ingeniero Forestal, Dr., Forestal Mininco S.A. Avenida Alemana 751, Los Ángeles, Chile. veronica.emhart@forestal.cmpc.cl

4. Ingeniero en Biotecnología Vegetal, Programa de Magister en Ciencias Biológicas, Departamento de Biología, Universidad de Chile, Las Palmeras 3425, Campus Juan Gómez Millas, Santiago, Chile. javier.saavedra.romero@gmail.com

Recebido para publicação em 26/05/2011 e aceito em 29/03/2012 


\section{INTRODUCCIÓN}

Eucalyptus globulus es usado principalmente para la producción de pulpa en diversas partes alrededor del mundo (WIMMER et al., 2002; LOPES et al., 2003; COSTA Y COLODETTE 2007; TONOLI et al., 2010). Algunas ventajas de la especie incluyen su rápido crecimiento $\mathrm{y}$ su buena adaptación a una gran variedad de climas y suelos (SANTELICES, 2005). A nivel mundial, la superficie plantada con Eucalyptus globulus corresponde aproximadamente a 20 millones de hectáreas (HUBBARD et al., 2010).

Desde el punto de vista del mejoramiento genético, diversas investigaciones se han llevado a cabo para cuantificar el grado de interacción genotipo-ambiente en varias especies del género Eucalyptus (LIMA et al., 2000; ROCHA et al., 2005; SILVA et al., 2006). El estudio de esta interacción permite evaluar la estabilidad de determinados genotipos en una gama de ambientes donde se desean introducir. La interacción surge cuando las entidades genéticas presentan diferentes respuestas dependiendo de las condiciones ambientales (SILVA et al., 2006). Los patrones de respuesta en diferentes ambientes involucran cambios en el ranking de genotipos y/o alteraciones en escala.

Varios esfuerzos en investigación con Eucalyptus se han centrado en el estudio de los genotipos bajo condiciones ambientales de riego suplementario. Por ejemplo, Hubbard et al. (2010) estudiaron cómo difiere el aporte de agua mensual y anual entre tratamientos de secano y regadío en plantaciones de Eucalyptus de rápido crecimiento. Por otra parte, White et al. (2000) examinaron la respuesta de árboles de Eucalyptus globulus bajo distintos niveles de riego, y su relación con el control de transpiración.

En Chile, según datos de INFOR (2009), las plantaciones de Eucalyptus globulus sobrepasan las 450 mil hectáreas, lo que representa cerca del $20 \%$ del total de plantaciones forestales del país. Se espera que estos valores aumenten en el tiempo debido al progreso realizado en las investigaciones relacionadas con la capacidad de resistencia a otras condiciones ambientales. En este contexto, Scott et al. (2002) investigaron el desarrollo de híbridos resistentes al frío de Eucalyptus gunnii x Eucalyptus globulus y Costa e Silva et al. (2008) analizaron la aclimatación/adaptación de rametos de Eucalyptus globulus, contrastando sus resultados con su resistencia a sequía.
Del punto de vista analítico, la inferencia Bayesiana ha sido usada crecientemente en innumerables campos de la ciencia, dado que ofrece la posibilidad de inferir acerca de los parámetros genéticos, utilizando los algoritmos de Monte Carlo-Cadenas de Markov (MCMC), generando medidas precisas sobre los estimadores a través de varianzas y errores estándares posteriores (SORENSEN y GIANOLA, 2002). El modelo de predicción Bayesiana de parámetros genéticos puede ser implementado usando el algoritmo de Gibbs; una variante de los algoritmos MCMC. De acuerdo con Cappa y Cantet (2006), en el ámbito de la estadística frecuentista (o clásica) no existe una contraparte equivalente a las distribuciones posteriores, por lo que las técnicas Bayesianas presentan una ventaja para la predicción de los parámetros genéticos.

Estudios relacionados con la predicción de valores genéticos y la estimación de componentes de varianza han confirmado que los procedimientos Bayesianos son equivalentes a los métodos de Máxima Verosimilitud Restringida (REML) y Mejor Predictor Lineal Insesgado (BLUP), siendo una alternativa efectiva en la evaluación genética de árboles forestales (CAPPA y CANTET, 2006; MORA et al., 2009). La correcta identificación de los individuos genéticamente superiores es crucial para los objetivos del mejoramiento genético, y requiere del conocimiento acerca del control genético de las características bajo selección (SANTOS et al., 2011). A su vez, los métodos Bayesianos han sido aplicados en la evaluación genética de árboles, que considera la medición de características complejas. Por ejemplo, Cané-Retamales et al. (2011) estimaron parámetros genéticos del florecimiento (característica medida en categorías) en Eucalyptus bajo condiciones ambientales áridas, utilizando un modelo umbral Bayesiano implementado por medio del algoritmo de Gibbs. De acuerdo con Stock y Distl (2010) los métodos MCMC permiten la implementación de modelos complejos (ej. umbrales (threshold) multivariados), los cuales son de especial interés en el mejoramiento genético (SANTOS et al., 2011).

El objetivo analítico del presente estudio fue evaluar genéticamente el crecimiento de 29 clones de Eucalyptus globulus de doce meses de edad, en dos sitios contrastantes en cuanto a la disponibilidad hídrica, utilizando para ello un análisis de predicción Bayesiana de parámetros genéticos, implementado por medio del algoritmo de Gibbs en un modelo de interacción clon-ambiente. 


\section{MATERIALES Y METODOS}

\section{Características del área de estudio}

El experimento se estableció en Octubre de 2008, en la región administrativa del Bío-Bío, Chile $\left(37^{\circ} 45^{\prime} \mathrm{S}\right.$ y $\left.72^{\circ} 18^{\prime} \mathrm{O}\right)$. El uso pasado del área seleccionada correspondió a un huerto semillero de Eucalyptus perteneciente a la empresa Forestal Mininco S.A. El clima del área es cálido y templado con estaciones secas y húmedas semejantes. La precipitación media anual es de $1200 \mathrm{~mm}$ con la mayoría de la precipitación durante los meses de invierno. La temperatura media anual es de $13,1{ }^{\circ} \mathrm{C}$ con una máxima media en verano de $18{ }^{\circ} \mathrm{C}$ y una mínima invernal de $6{ }^{\circ} \mathrm{C}$. Los suelos corresponden a la serie Collipulli, Alfisoles rojo arcillosos desarrollados de cenizas volcánicas antiguas altamente meteorizadas que han formado suelos rojo arcillosos profundos sobre terrenos ondulados en el valle central de Chile (INIA, 1985). El ensayo consideró la evaluación de genotipos de Eucalyptus globulus con y sin suplemento de riego.

\section{Material genético y diseño experimental}

Se consideró la evaluación genética de 29 clones elite de Eucalyptus globulus. El ensayo consideró un diseño en bloques completamente al azar, con tres bloques, y cuatro rametos por clon. Las plantas seleccionadas se obtuvieron a partir de estacas enraizadas con siete meses de viverización y considerando tamaños homogéneos. Las plantas se establecieron con fecha 28 de Octubre de 2008 por medio de cultivo con pala plantadora en casillas de $20 \times 20 \times 20 \mathrm{~cm}$ de profundidad. Las plantas se fertilizaron en la casilla de plantación con una dosis de $30 \mathrm{~g}$ de Basacote Plus 9M (Compo S.A.) por planta con una composición de $16 \% \mathrm{~N}+8 \% \mathrm{P} 2 \mathrm{O} 5+$ $12 \% \mathrm{~K} 20+2 \% \mathrm{MgO}+5 \% \mathrm{SO} 2+$ micro-elementos. A efectos de asegurar las mejores condiciones de homogeneidad para expresión de las respuestas de cada genotipo, se aplicaron controles de maleza sucesivos en cada área de estudio hasta el cierre de copas en parcelas con y sin riego.

El tratamiento de riego se consideró sólo durante el período estival dada la alta capacidad de almacenamiento de agua del suelo y la textura arcillosa del mismo. Las cantidades de riego diario aplicados se estimaron considerando la reposición de pérdida de la bandeja de evaporación, pero con un límite máximo de reposición de $3,2 \mathrm{~mm} \cdot$ día $^{-1}$. Los montos mensuales acumulados de riego fueron $54,4 \mathrm{~mm}$ en Octubre 2008, 86,4 $\mathrm{mm}$ en Noviembre
2008, 99,2 mm en los meses de Diciembre 2008 y Enero 2009, 82,4 mm en Febrero 2009 y 86,4 mm en Marzo 2009. La aplicación de riego se efectuó ocupando un sistema de cinta con goteros aledaños a cada planta sobre superficie asegurando una mejor disponibilidad de agua para la planta. Las condiciones de disponibilidad de agua en el suelo se evaluaron quincenalmente por medio de tres sensores de TDR localizados entre $0-15$ y $15-30 \mathrm{~cm}$ de profundidad en cada bloque y de $0-20 \mathrm{~cm}$ horariamente vía sensores continuos de humedad ECHO-EC20 Soil Moisture Probe (DECAGON Devices).

A los doce meses de edad, los clones se evaluaron en función de las siguientes características de crecimiento: a) altura total de las plantas; b) diámetro basal; y c) área basal.

\section{Estimación de parámetros genéticos}

El modelo lineal bi-carácter para ambos ambientes (MORA et al., 2010; CANÉ-RETAMALES et al., 2011) que representó los datos del experimento fue el siguiente:

$$
\begin{aligned}
& y=X \beta+Z g+P p+\varepsilon \\
& y=\left[\begin{array}{l}
y_{1} \\
y_{2}
\end{array}\right]=\left[\begin{array}{cc}
X_{1} & 0 \\
0 & X_{2}
\end{array}\right]\left[\begin{array}{l}
\beta_{1} \\
\beta_{2}
\end{array}\right]+\left[\begin{array}{cc}
Z_{1} & 0 \\
0 & Z_{2}
\end{array}\right]\left[\begin{array}{l}
g_{1} \\
g_{2}
\end{array}\right]+\left[\begin{array}{cc}
P_{1} & 0 \\
0 & P_{2}
\end{array}\right]\left[\begin{array}{l}
p_{1} \\
p_{2}
\end{array}\right]+\left[\begin{array}{l}
\varepsilon_{1} \\
\varepsilon_{2}
\end{array}\right]
\end{aligned}
$$

donde $y_{1}$ y $y_{2}$ son los vectores de observaciones para una determinada característica en los ambientes 1 y 2 respectivamente; considerando el efecto de interacción clon-ambiente. $X$ es la matriz de incidencia para el vector $\beta$, el cual representa un vector con los efectos de ambiente y bloque dentro de ambiente. $Z$ es la matriz de incidencia del vector de efectos genotípicos $g$. $P$ es la matriz de incidencia del vector de efectos debido a la parcela $p$. $\varepsilon$ es el vector de residuos. Los componentes de covarianza y varianza, heredabilidad en sentido amplio, y la predicción de los valores genéticos se investigaron por medio del uso de inferencia Bayesiana, implementada por medio del algoritmo de Gibbs.

Se calculó la heredabilidad individual en sentido amplio para las características de crecimiento, usando la siguiente expresión:

$$
H^{2}=\frac{\sigma_{g}^{2}}{\sigma_{g}^{2}+\sigma_{p}^{2}+\sigma_{e}^{2}}
$$

donde $\sigma_{g}^{2}$ corresponde a la varianza genotípica entre clones; $\sigma^{2}$ a la varianza dentro de parcelas; $\sigma_{\mathrm{e}}^{2}$ varianza residual. 
La correlación genética entre ambientes para cada característica, se estimó usando la siguiente expresión:

$$
\Gamma=\frac{\sigma_{g_{x y}}}{\sqrt{\sigma_{g_{x}}^{2} \cdot \sigma_{g_{y}}^{2}}}
$$

donde $\sigma_{\mathrm{g}_{\mathrm{xy}}}$ corresponde a la covarianza clonal entre los ambientes con riego y sin riego, $\mathrm{x}$ e y respectivamente. $\sigma_{\mathrm{g}_{\mathrm{x}}}^{2}$ y $\sigma_{\mathrm{g}_{\mathrm{y}}}^{2}$ son los componentes de varianza clonal para cada ambiente.

De acuerdo con el procedimiento bayesiano, las matrices de varianza genética, procedencias y residual tienen las siguientes distribuciones $a$ priori, respectivamente:

$$
G_{*} \sim I W\left(G_{0}, v_{g}\right) P_{*} \sim I W\left(P_{0}, v_{p}\right) \quad R_{*} \sim I W\left(R_{0}, v_{e}\right)
$$

o bien:

$$
\begin{aligned}
& f\left(G_{*} \mid G_{0}, v_{g}\right) \propto(G)^{-\frac{1}{2}\left(v_{g}+m_{g}+1\right)} \cdot e^{-\frac{1}{2} \operatorname{tr}\left(G_{*}^{-1} G_{0}^{-1}\right)} \\
& f\left(P_{*} \mid P_{0}, v_{p}\right) \propto(P)^{-\frac{1}{2}\left(v_{p}+m_{p}+1\right)} \cdot e^{-\frac{1}{2} \operatorname{tr}\left(P_{*}^{-1} P_{0}^{-1}\right)} \\
& f\left(R_{*} \mid R_{0}, v_{r}\right) \propto(R)^{-\frac{1}{2}\left(v_{r}+m_{r}+1\right)} \cdot e^{-\frac{1}{2} \operatorname{tr}\left(R_{*}^{-1} R_{0}^{-1}\right)}
\end{aligned}
$$

donde, $G_{0}, P_{0}$ y $R_{0}$ son las matrices con los valores a priori de los componentes de (co) varianza. $v_{-}$es el grado de credibilidad bayesiano y $m_{-}$es la dimensión de la matriz parámetro escalar. La distribución a priori de $\beta$ se consideró uniforme (constante); y los vectores $g$ y $p$ normalmente distribuidos:

$$
\begin{aligned}
& f(\beta) \sim \text { Uniforme } \\
& f\left(y \mid \beta, g, p, G_{*}, P_{*}\right) \sim N\left(X \beta+Z g+P p, R_{*}\right) \\
& f\left(g \mid G_{*}\right) \sim N\left(0, G_{*}\right)
\end{aligned}
$$

El procedimiento Bayesiano se implementó usando el programa MTGSAM (Multi-trait Gibbs Sampling for Animal Models) (VAN-TASSELL y VAN-VLECK, 1996). Se chequeó la convergencia de las cadenas de Gibbs usando el test de Heidelberger y Welch (1983), calculado en el programa $\mathrm{R}$ con la librería CODA (Convergence Diagnosis and Output Analysis).

\section{RESULTADOS Y DISCUSIÓN}

Las iteraciones de Gauss-Seidel, realizadas previamente a la conformación de la cadena de Gibbs, convergieron en la iteración 21, 30 y 50, para la altura, diámetro y área basal, respectivamente, y con un criterio de convergencia de $0,65 \mathrm{E}-9,0,8 \mathrm{E}-9 \mathrm{y}$ 0,88 . Se confirmó la convergencia de las cadenas de Gibbs utilizando 500 mil muestras para cada cadena, incluyendo el periodo de descarte o burn-in, el cual fue considerado en 100 mil muestras. Los resultados se registraron a cada 40 iteraciones para evitar auto-correlación en cada cadena.

En la Tabla I se muestran los resultados de la distribución a posteriori (AP) de la heredabilidad individual en sentido amplio para cada característica, determinada por medio de la media, mediana, moda e intervalo de credibilidad AP. Los valores de

TABLA 1: Caracterización de la distribución a posteriori de la heredabilidad individual en sentido amplio, determinada por medio de procedimientos Bayesianos en 29 clones de Eucalyptus globulus.

TABLE 1: Posterior distribution for the heritability determined by Bayesian procedures in 29 clones of Eucalyptus globulus.

\begin{tabular}{lcccccc}
\hline & \multicolumn{2}{c}{ Altura } & \multicolumn{2}{c}{ Diámetro } & \multicolumn{2}{c}{ Área basal } \\
\cline { 2 - 7 } & $\mathrm{R}$ & $\mathrm{SR}$ & $\mathrm{R}$ & $\mathrm{SR}$ & $\mathrm{R}$ & $\mathrm{SR}$ \\
\hline Media & 0,425 & 0,155 & 0,355 & 0,228 & 0,402 & 0,176 \\
Mediana & 0,426 & 0,147 & 0,354 & 0,222 & 0,399 & 0,169 \\
Moda & 0,413 & 0,123 & 0,361 & 0,213 & 0,393 & 0,152 \\
\hline DE & 0,094 & 0,070 & 0,086 & 0,064 & 0,086 & 0,062 \\
\hline IC & & & & & & \\
Inferior & 0,269 & 0,057 & 0,216 & 0,135 & 0,263 & 0,085 \\
Superior & 0,579 & 0,283 & 0,500 & 0,347 & 0,548 & 0,288 \\
\hline
\end{tabular}

R y SR: ambientes con riego y sin riego, respectivamente; DE: Desviación estándar; IC: intervalo de credibilidad Bayesiano (95\% de probabilidad) 
la heredabilidad fueron altos para la situación con riego suplementario, en tanto que para el ambiente sin riego, las heredabilidades fueron significativamente más bajas; los intervalos de credibilidad Bayesiano (95\% de probabilidad) para la situación sin riego, no incluyeron los valores puntuales AP de la heredabilidad para la situación con riego, en todas las características evaluadas, por lo tanto se afirma que las heredabilidades entre los ambientes son distintas del punto de vista estadístico (95\% de probabilidad). Los valores de heredabilidad fueron similares entre las características de crecimiento, lo cual se evidenció en ambos ambientes. Los valores de la moda de las heredabilidades fueron similares a los valores de las estimaciones puntuales del promedio, evidenciando una relativa simetría en las distribuciones AP de dicho parámetro genético.

Los valores de heredabilidad están en concordancia con lo encontrado por Ignacio-Sánchez et al. (2005) en un ensayo clonal de Eucalyptus urophylla de tres años y medio establecido en Veracruz, México, cuyas magnitudes de heredabilidad individual para las características de altura total, diámetro a la altura de pecho (DAP) y volumen del tronco fluctuaron entre 0,26 y 0,34 , al primer año de edad. En una población base de cinco ensayos de procedencia/progenie de polinización abierta de Eucalyptus viminalis, establecidos en Argentina, Cappa et al. (2010) encontraron moderados valores de heredabilidad en sentido estricto para el crecimiento $(0,17-0,27)$. En Portugal, Costa e Silva et al. (2009) encontraron un moderado control genético aditivo del DAP, en una población de Eucalyptus globulus generada de cruzas controladas entre hermanos completos, variando de 0,11 a 0,35. A su vez, Hunde et al. (2007), en un ensayo de procedencia/progenie de Eucalyptus globulus establecido en Etiopía, encontraron moderadas heredabilidades individuales - dentro de procedencias para la altura $(0,20)$ y el diámetro $(0,16)$.

En ensayos de polinización abierta de Eucalyptus globulus, López et al. (2002) encontra- ron heredabilidades del crecimiento de 0,21 y 0,23 (promedio), cuyos valores son considerados como valores típicos para el diámetro y la altura, respectivamente, de acuerdo con Cappa et al. (2010). En la situación con riego del presente estudio, se evidencian valores más altos de la heredabilidad de los reportados por López et al. (2002), y en particular para la altura, en donde el intervalo de credibilidad Bayesiano no incluye el valor promedio encontrado por los autores. Este resultado podría estar asociado a las condiciones de riego suplementario que han sido sometidas las plantas.

En la Tabla 2 se muestran los resultados del análisis Bayesiano para la distribución AP de la correlación genética entre ambientes para cada característica, descritas por medio de la media, mediana, moda e intervalo de credibilidad ( $95 \%$ de probabilidad). De acuerdo con Sykes et al. (2006) un valor de correlación entre ambientes cercano a 1 indica escasa interacción, mientras que un valor bajo (o no significativamente diferente de cero) indica la existencia significativa de interacción, y el ranking puede ser diferente en cada sitio.

Una ventaja de los procedimientos Bayesianos es la posibilidad de usar las regiones de credibilidad como una alternativa de estimación de parámetros genéticos, los cuales son obtenidos directamente de la distribución AP (MORA et al., 2009; WRIGHT et al., 2000; SANTOS et al., 2011). De acuerdo a este principio, las correlaciones genéticas del presente estudio fueron estadísticamente diferentes de cero (intervalo de credibilidad Bayesiano de $95 \%$ de probabilidad), indicando que la interacción no sería significativa. Los clones se mantienen relativamente estables en los ambientes evaluados. Estos resultados son coincidentes con los valores del coeficiente de correlación de Spearman, calculado entre los rankings de cada ambiente y característica. Los valores de Spearman fueron 0,77, 0,66 y 0,80 para la altura, diámetro y área basal, respectivamente $(\mathrm{P}<0,01)$.

TABLA 2: Caracterización de la distribución a posteriori de la correlación genética entre ambientes, determinada por procedimientos Bayesianos en 29 clones de Eucalyptus globulus.

TABLE 2: Posterior distribution for the genetic correlation between environments determined by Bayesian procedures in 29 clones of Eucalyptus globulus.

\begin{tabular}{lccccc}
\hline Característica & Promedio & Mediana & Moda & DE & IC \\
\hline Altura & 0,558 & 0,594 & 0,704 & 0,234 & $(0,120-0,871)$ \\
Diámetro & 0,560 & 0,583 & 0,651 & 0,185 & $(0,217-0,818)$ \\
Área basal & 0,715 & 0,750 & 0,819 & 0,167 & $(0,388-0,918)$ \\
\hline
\end{tabular}

DE: Desviación estándar; IC: intervalo de credibilidad Bayesiano ( $95 \%$ de probabilidad) 
La Figura 1 muestra un gráfico de dispersión con los valores genéticos de los 29 clones de Eucalyptus globulus, para cada ambiente y característica. La línea recta confirma el alto grado de asociación genética entre los ambientes considerados en el ensayo. A pesar del bajo número de genotipos evaluados, la moderada variabilidad genética encontrada en este estudio, representada por medio de la heredabilidad en sentido amplio, permite un margen de selección de los clones de mejor desempeño. En efecto, la ganancia genética considerando la selección de los mejores clones (intensidad de selección de 20,7\%), de acuerdo a su valor genético, varió de $8 \%$ (altura) a $23 \%$ (área basal).

Mora y Perret (2007) resumieron la utilidad del enfoque bayesiano en la teoría del mejoramiento forestal y en la evaluación genética de árboles de Eucalyptus. Del punto de vista de la evaluación genética, el procedimiento Bayesiano, usando diferentes métodos MCMC (ej. algoritmo de Gibbs), es indicado como una alternativa al método de estimación/predicción de modelos mixtos (CAPPA y CANTET 2006; GONÇALVES-VIDIGAL et al., 2008; CANÉ-RETAMALES et al., 2011; RESENDE, 2002; MORA y PERRET, 2007). El procedimiento bayesiano lleva en consideración la variabilidad existente en todos los parámetros del modelo en el sentido de que es posible caracterizarlos a través de la moda, mediana o el promedio de la distribución AP de dicho parámetro, así como la obtención de intervalos de confianza (llamadas de regiones de credibilidad en el caso bayesiano) tanto para los parámetros como para la predicción de los efectos genéticos. En el presente estudio, el bajo número de clones (genotipos) evaluados, podría ser un factor limitante del punto de vista de la precisión de los parámetros genéticos (RESENDE, 2002), sin embargo, diversos estudios (GONÇALVESVIDIGAL et al., 2008; MORA et al., 2009) han confirmado las ventajas de la inferencia bayesiana cuando se tiene un bajo número de genotipos.

\section{CONCLUSIONES}

En el presente estudio, las características de crecimiento son altamente heredables en la situación con riego suplementario. En el sitio sin irrigación los genotipos muestran significativamente menos variación genética.

La existencia de un nivel significativo de varianza genética en las variables de crecimiento de los clones de Eucalyptus globulus en el primer año de edad, sugiere la realización de estudios posteriores que permitan determinar la tendencia del control genético en el tiempo.
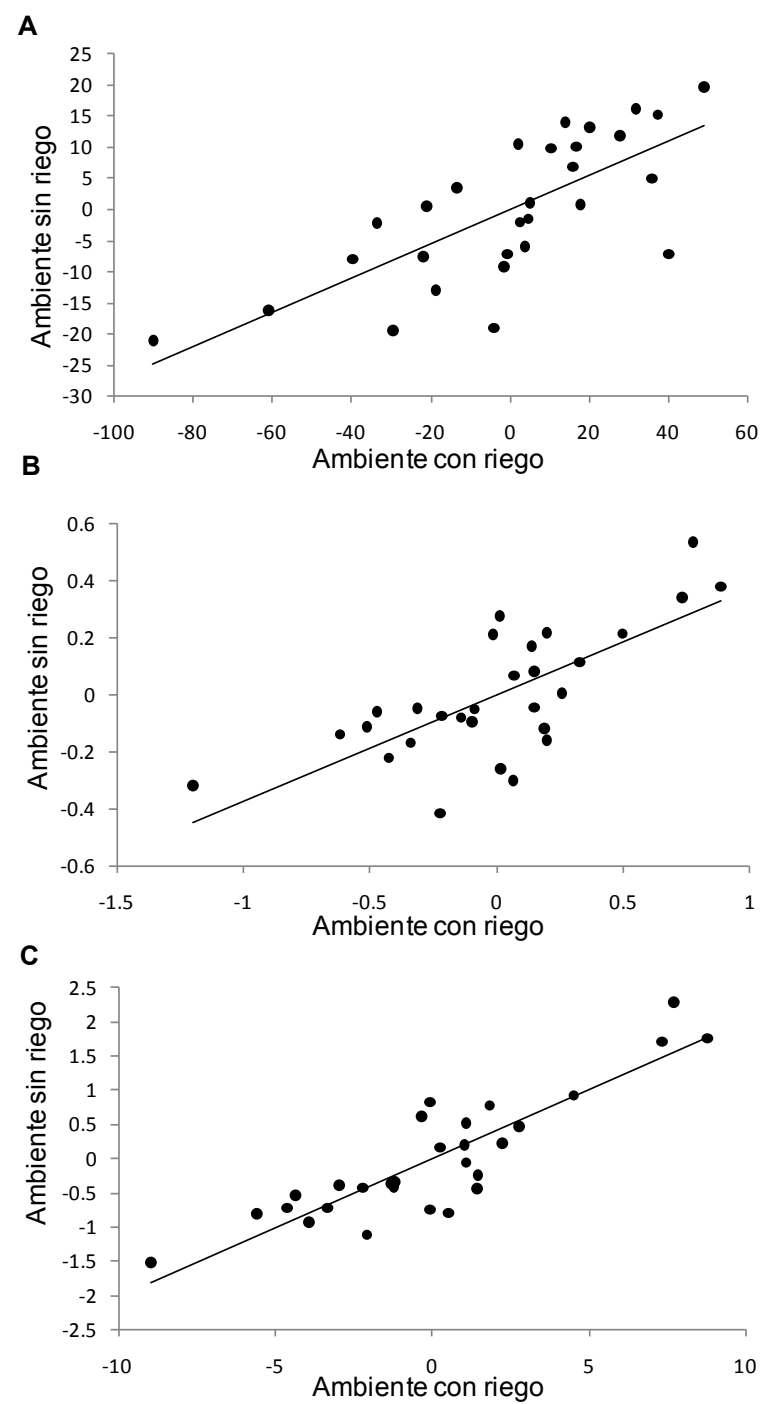

FIGURA 1: Valores genéticos de 29 clones de Eucalyptus globulus, predichos por medio de un análisis Bayesiano, vía algoritmo de Gibbs, para cada ambiente y cada característica de crecimiento, A: altura, B: diámetro basal, y C: área basal. La línea recta indica el grado de asociación genética entre ambientes.

FIGURE 1: Breeding values for 29 clones of Eucalyptus globulus, which were predicted by using Bayesian methods via Gibbs sampling, for each site and growth trait, A: height, B: basal diameter, and $\mathrm{C}$ : sectional area. The lines indicate the degree of genetic association between environments. 


\section{AGRADECIMIENTOS}

La presente investigación fue financiada por FONDECYT, proyectos 11090129 y 1085093. Agradecimientos a la empresa Forestal MININCO S.A. por proveer el material genético del presente estudio.

\section{REFERENCIAS BIBLIOGRÁFICAS}

CANE-RETAMALES, C. et al. Bayesian threshold analysis of breeding values, genetic correlation and heritability of flowering intensity in Eucalyptus cladocalyx under arid conditions. Euphytica, Wageningen, v. 178, n. 2. p 177-183, Mar. 2011.

CAPPA, E. et al. Provenance variation and genetic parameters of Eucalyptus viminalis in Argentina. Tree Genetics \& Genomes, Davis, v. 6, p. 981-994, June 2010.

CAPPA, E.; CANTET, R. Bayesian inference for normal multiple-trait individual-tree models with missing records via full conjugate Gibbs. Canadian Journal of Forest Research, Ottawa, v. 36, n. 5, p. 1276-1285, May, 2006.

COSTA E SILVA, F. et al. Acclimation to shortterm low temperatures in two Eucalyptus globulus clones with contrasting drought resistance. Tree Physiology, Victoria, v. 29, n.1, p. 77-86, Sept. 2008.

COSTA E SILVA, F. et al. Genetic parameters for growth, wood density and pulp yield in Eucalyptus globulus. Tree Genetics \& Genomes, Davis, v. 5, p. 291-305, Sept. 2009.

COSTA, M.; COLODETTE, J. The impact of kappa number composition on Eucalyptus kraft pulp bleachability. Brazilian Journal of Chemical Engineering, v. 24, n.1, p 61-71, Jan./Mar. 2007. GONÇALVES-VIDIGAL, M. C. et al. Heritability of quantitative traits in segregating common bean families using a Bayesian approach. Euphytica, Wageningen, v. 164, p. 551-560, July 2008.

HEIDELBERGER, P.; WELCH, P.D. Simulation run length control in the presence of an initial transient. Opns Res, v. 31, p. 1109-44, 1983.

HUBBARD, R. et al. Effects of irrigation on water use and water use efficiency in two fast growing Eucalyptus plantations. Forest Ecology and Management, Amsterdam, v. 259, p. 1714-1721, 2010.

HUNDE, T. et al. Genetic variation in survival and growth of Eucalyptus globulus Ssp. globulus in Ethiopia. Australian Forestry, Queen Victoria.
Yarralumla, v. 70, n. 1, p. 48-52, Mar 2007.

IGNACIO-SÁNCHEZ, E. et al. Genetic parameters for growth and wood density in juvenile Eucalyptus urophylla S. T. Blake. Agrociencia, Montecillo, v. 4, p. 469-479, 2005.

INFOR. Inventario continuo de bosques nativos y actualizaciones de plantaciones forestales. Disponível em $<$ (http://www.infor.gov.cl/es/ component/docman/doc_details/41-inventariocontinuo-de-bosques-nativos-y-actualizacionesde-plantacionesforestales.html)> Acesso em: 25 de maio de 2011.

INIA. Suelos volcánicos de Chile. Santiago do Chile: Ministerio de Agricultura, 1985.

LIMA, J. et al. Genotype-environment interaction in wood basic density of Eucalyptus clones. Wood Science and Technology, Berlin, v. 34, n. 3, p. 197 206, 2000.

LOPES, E. et al. Application of life cycle assessment to the Portuguese pulp and paper industry. Journal of Cleaner Production, v. 11, p. 51-59, ene. 2003. LOPEZ, G. A. et al. Genetic variation and inter-trait correlations in Eucalyptus globulus base population trials in Argentina. Forest Gen, v. 9, n. 3, p. 223237, Sept. 2002.

MORA, F. et al. El análisis bayesiano y la precisión de los valores de la heredabilidad en especies perennes. Ciência Florestal, Santa Maria, v. 19, n. 4, p. 345351, out./dez. 2009.

MORA, F. et al. Genetic parameters of growth and survival in Acacia saligna shrubs. Ciencia e Investigación Agraria, v. 37, n. 2, p. 5-14, May 2010.

MORA, F.; PERRET, S. Aplicación de técnicas bayesianas en el análisis genético de árboles forestales. Bosque, Valdivia, v. 28, n. 3, p. 198-206, 2007.

RESENDE, M. D. V. Genética biométrica e estatística no melhoramento de plantas perenes. Brasília: EMBRAPA Informação Tecnológica, 2002. $975 \mathrm{p}$.

ROCHA, R. B. et al. Avaliação do método centróide para estudo de adaptabilidade ao ambiente de clones de Eucalyptus grandis. Ciência Florestal, Santa María, v. 15, n. 3, p. 255-266, 2005.

SANTELICES, R. Desarrollo de una plantación de Eucalyptus globulus establecida en primavera con diferentes tratamientos de riego. Bosque, Valdivia, v. 26, n. 3, p. 105-112, feb. 2005.

SANTOS, A. I. et al. Bayesian genetic parameters for body weight and survival of Nile tilapia farmed in Brazil. Pesquisa Agropecuária Brasileira, 
Brasília, v. 46, n. 1, p. 33-43, jan. 2011.

SCOTT, S. et al. Possum browsing-the downside to a eucalypt hybrid developed for frost tolerance in plantation forestry. Forest Ecology and Management, Amsterdam, v. 157, p. 231-245. 2002. SILVA, J. C. et al. Genotype by environment interaction for growth of Eucalyptus globulus in Australia. Tree Genetics \& Genomes, Davis, v. 2, n. 2, p. 61-75, Feb. 2006.

SORENSEN, D.; GIANOLA, D. Likelihood, Bayesian, and MCMC methods in quantitative genetics. New York: Springer-Verlag, 2002.

STOCK, K. F.; DISTL, O. Simulation study on the effects of excluding offspring information for genetic evaluation versus using genomic markers for selection in dog breeding. Journal of Animal Breeding and Genetics, Jokioinen, v. 127, n. 1, p. 42-52, June 2009.

SYKES, R. et al. Genetic Variation and genotype by environment interactions of juvenile wood chemical properties in Pinus taeda L. Annals of Forest Science, Les Ulis, v. 63, p. 897-904, Feb. 2006.
TONOLI, G. et al. Eucalyptus pulp fibres as alternative reinforcement to engineered cementbased composites. Industrial Crops and Products, v. 31, n.2, p. 225-232, mar. 2010.

VAN-TASSELL, C. P.; VAN-VLECK, L. D. Multiple-trait Gibbs sampler for animal models: flexible programs for Bayesian and likelihood-based (co)variance component inference. Journal of Animal Science, Champaign, v. 74, p. 2586-2597, 1996.

WHITE, D. et al. Control of transpiration in a irrigated Eucalyptus globulus Labill. Plantation. Plant, Cell and Environment, Oxford, v. 23, n. 2, p. 123-134, Dec. 2000.

WIMMER, R. et al. Direct effects of wood characteristics on pulp and handsheet properties of Eucalyptus globulus. Holzforschung, Berlin, v. 56, n. 3, p. 244-252. 2002.

WRIGHT, D. et al. Comparing traditional and Bayesian analyses of selection experiments in animal breeding. Journal of Agricultural, Biological, and Environmental Statistics, Alexandria, v. 5, n. 2, p. 240-256, June 2000. 